\title{
Spectrally controlled source for interferometric measurements of multiple surface cavities
}

Chase Salsbury, Jan Posthumus, Artur Olszak

Chase Salsbury, Jan Posthumus, Artur Olszak, "Spectrally controlled source for interferometric measurements of multiple surface cavities," Proc. SPIE 10829, Fifth European Seminar on Precision Optics Manufacturing, 108290C (7 August 2018); doi: 10.1117/12.2318641

Event: Fifth European Seminar on Precision Optics Manufacturing, 2018, Teisnach, Germany 


\title{
Spectrally controlled source for interferometric measurements of multiple surface cavities
}

\author{
Chase Salsbury ${ }^{\mathrm{a}, \mathrm{c}}$, Jan Posthumus ${ }^{\mathrm{a}, \mathrm{b}}$, and Artur Olszak ${ }^{\mathrm{a}}$ \\ a Äpre Instruments, LLC, 2440 W Ruthrauff Rd 100, Tucson, AZ, 85705 \\ ${ }^{\mathrm{b}}$ LaserPeak, GmbH, Arnethgasse 80,1160 Wien, Austria \\ ${ }^{c}$ College of Optical Sciences, University of Arizona, 1630 E University Blvd, Tucson, AZ, 85716
}

\begin{abstract}
We present a new light source capable of locating interference fringes at an adjustable distance from the interferometer. The spectrum is electronically controlled in such a way that the fringes are limited to only one of the surfaces of the optics under test. With the new source it is straightforward, for example, to measure the parallel surfaces of thin glass plates and multiple surface cavities. Existing interferometers, as well as older systems, can be upgraded with this source.

Traditional methods of interferometry are widely used and accepted for simple measurement configurations, but measurement accuracy can decrease rapidly with increasing measurement complexity. For example, coherent interferometry struggles to achieve accurate and repeatable results with the presence of any additional feedback surface in the measurement cavity due to temporally coherent back reflections. Conversely, incoherent interferometers can isolate single surfaces for measurement but require more complex interferometer system designs. As a result, many of these systems are limited in their dynamic range of measurable cavity sizes and present considerable difficulties in the alignment process, increasing total measurement time. Both methods are inherently restricted by the intrinsic properties of their respective source.

Spectrally controlled interferometry (SCI) is a source driven method which inherits many advantages from both coherent and incoherent interferometry while evading typical limitations. The sources spectral properties are manipulated to produce a tunable coherence function in measurement space which allows control over the coherence envelope width, the fringe location, and the fringe phase. With this source realization, a host of measurement advantages which simplify measurement complexity and reduce total measurement time becomes available. One major application is the extinction of extraneous surface back reflections. Without any mechanical translation, realignment, or traditional piezoelectric transducers, front and back surfaces of planar optics can be isolated independently and complete phase shifting interferometric (PSI) measurements can be taken. Furthermore, because all control parameters are implemented at the source level, the spectrally controlled source is a good candidate for upgrading existing interferometer systems.
\end{abstract}

In this paper, we present the theoretical background for this source and the implications of the method. Additionally, a multiple surface cavity measurement is provided as a means of demonstrating the spectrally controlled sources capability to isolate individual cavities from detrimental back reflections across a large dynamic range of measurable cavity sizes without mechanical realignment. A discussion of the implementation benefits and practical details will be included. Limitations and comparisons to alternative methods will be addressed, as well.

Keywords: interferomtery, metrology, spectrally controlled interferometry, SCI, phase shifting,white light interferometry,laser interferometry,coherence

Further author information: (Send correspondence to Chase Salsbury)

Chase Salsbury: E-mail: csalsbury@apre-inst.com

Fifth European Seminar on Precision Optics Manufacturing, edited by Rolf Rascher, Christian Schopf, Proc. of SPIE Vol. 10829, 108290C · (C) 2018 SPIE

CCC code: $0277-786 \mathrm{X} / 18 / \$ 18 \cdot$ doi: $10.1117 / 12.2318641$

Proc. of SPIE Vol. 10829 108290C-1 


\section{INTRODUCTION}

\subsection{Optical Interferometry Methods}

Optical interferometry is the dominant method for optical surface characterization and testing due to its level of accuracy and the relatively simple system design. The measurement of planar optical components (windows, filters, bulk glass substrates, etc) make up a large portion of the applications for interferometers, but offer significant challenges that often require complicated or time consuming set up practices and often cannot be measured by traditional methods at all. While there are countless numbers of interferometer configurations, general modes of interferometry can be organized into two main categories depending on the type of light source used.

Coherent, or laser, interferometry is the most common commercial method for optical surface measurement and characterization. Laser sources have very long coherence lengths (often on the order of meters) due to the narrow extent of their optical frequency line-width. Because interference will still occur for large values of optical path difference (OPD), laser interferometers are most commonly configured as Fizeau interferometers. ${ }^{1}$ These systems are unbalanced interferometers and have the advantage of operating in a common path and benefit from aberration cancellation in the absence of retrace error. ${ }^{2}$

As an additional benefit, there is very little constraint on the axial alignment of the optical surface under test in order to produce high contrast interference fringes. However, this additional degree of freedom also presents challenges for surface isolation. Every optical surface within the coherence length of the laser source, will produce a temporally coherent back reflection at the detector plane. For parallel or quasi-parallel planar optical components, there are three optical surfaces within the coherence length when a transmission flat is used for reference. The presence of more than two temporally coherent back reflections, produces interference fringe pattern ambiguity and can reduce measurement quality and in most cases impede measurement altogether. ${ }^{3}$

While there are various software and phase unwrappping approaches which attempt to separate these entangled fringe patterns into their individual surface contributions, ${ }^{4,5}$ these methods are often limited to a finite number of additional surfaces or may require more complex phase shifting combinations or phase map processing. In many cases, the optic under test is modified to remove or prevent unwanted back reflections from the additional surfaces by the means of masking tape, Vaseline, or in more acute cases anti-reflection coating. The application and removal of these anti-reflection materials increases the risk of damage to these optics and comes at the expense of time.

The other common mode of interferometry is incoherent, or white-light, interferometry in which a broadband source with finite extent of the optical frequency line-width is used. For these types of sources, high contrast interference occurs only at the condition of zero OPD and as a result a balanced interferometer, such as the Twyman-Green, for example, must be used for these systems. ${ }^{6}$ The Twyman-Green interferometer does not benefit from common path aberration cancellation and is more sensitive to internal aberrations and wavefront distortion. However, through the use of mechanical path length matching, this configuration approach is capable of limiting coherent interference to just a single surface of an optical component under test, removing parasitic back reflections which cause fringe ambiguity at the detector. Instead, contributions from secondary surfaces are incoherent and add only background intensity. ${ }^{6}$

While incoherent interferometry addresses the issue of surface isolation without modification to the optical component under test, these systems require high precision mechanical translation stages to achieve repeatable and stable path length matching. To achieve comparable dynamic range of measurement space to the Fizeau interferometer, the complexity, cost, and mechanical footprint of these systems can become significant. From a practical standpoint, these systems can be difficult to use due to the narrow extent of the region of high contrast fringes. The required time to complete the alignment procedure is often drastically increased because the operator must find where the fringes are located in space to the accuracy of microns and is left with no feedback mechanism outside of this region.

Fourier transform phase shifting interferometry, ${ }^{7,8}$ is an alternative interferometric approach to multiple surface measurement. This is a compound method which combines a precise wavelength shifting laser source with additional Fourier transform processing to identify and isolate individual surface contributions. However, this approach requires high resolution wavelength shifting for large cavities and large dynamic range wavelength 
shifting for small cavities which can be difficult to achieve for commercially available tunable lasers. This method requires longer acquisiton times to allow scanning of the mean wavelength, making it more susceptible to vibration and other environmental factors.

A novel, spectrally controlled light source offers a flexible and convenient solution to many of the associated limitations with the traditional coherent and incoherent methods of interferometry. As its name implies, the spectral distribution of a broadband source is manipulated in order to produce localized fringes at adjustable distances for single surface isolation. These localized fringes are free from the requirement of zero OPD and are capable of being phase shifted for complete and accurate phase measurement, allowing integration into Fizeau or any equivalent common-path interferometer architectures. Furthermore, fringe location and phase are both controlled electronically, removing the need for bulky and expensive translation stages dedicated to path length matching or piezoelectric transducers typically employed for phase shifting. ${ }^{9-11}$ Previous methods for producing white light Fizeau interferometers have resulted in a comb-like distribution of high contrast interference fringes ${ }^{12-16}$ which does not completely address the problem of fringe ambiguity and resulted in fringes would still required mechanical translation for phase shifting.

\subsection{Optical Measurement and Characterization}

Surface figure and refractive index homogeneity are among the most common measurements of optical components for manufacturers. Surface figure refers to the shape of the surface under test in relation to a known reference surface such as a transmission flat and can be measured directly with traditional phase shifting interferometry (PSI) techniques. ${ }^{2}$ The surface shape is responsible for the majority of wavefront distortion and is often specified in terms of Peak-Valley or Root Mean Square (RMS) surface shape metrics.

Refractive index homogeneity measurement is an assessment of the local fluctuations in refractive index relative to the nominal bulk refractive index. This characterization is more difficult to measure directly as the effects from refractive index are combined together with effects from surface deformations of the optical component under test. ${ }^{17}$ In the traditional method of homogeneity measurement, four measurements are required with a four surface cavity to remove the contributions from surface deformation, shown in Table $1 .{ }^{18}$

Table 1. Required measurements and their descriptions for traditional homogeneity measurements.

\begin{tabular}{|l|l|}
\hline M1: Front surface & Transmission flat and front surface of optic under test \\
\hline M2: Back surface & $\begin{array}{l}\text { Transmission flat and back surface of optic under test, } \\
\text { measured through the optic under test }\end{array}$ \\
\hline M3: Transmitted wavefront & $\begin{array}{l}\text { Transmission flat and reference flat, } \\
\text { measured through the optic under test }\end{array}$ \\
\hline M4: Empty cavity & $\begin{array}{l}\text { Transmission flat and reference flat, } \\
\text { measured with the optic under test removed from the cavity }\end{array}$ \\
\hline
\end{tabular}

With this series of measurements, from, refractive index homogeneity of the material can be calculated based on the phase maps relationships in (1). ${ }^{18}$

$$
\Delta n(x, y)=\frac{1}{2 t}\left[n_{o}\left(M_{3}(x, y)-M_{4}(x, y)\right)-\left(n_{0}-1\right)\left(M_{2}(x, y)-M_{1}(x, y)\right)\right]
$$

The combination of multiple surface measurements allows the effective removal of contributions from surface shape and deformations, but depends on the nominal bulk refractive index, $n_{0}$, and thickness, $t$, of the material which must be known by the operator. Changing the thickness in the calculation will only scale the magnitude of the result, but changing the refractive index will have more serious consequences as the phase maps are scaled by refractive index. It is important that these parameters are known with a reasonable degree of certainty. ${ }^{18}$ 


\section{THEORY}

The difference in operation between laser and white light interferometers is due to the intrinsic characteristics of the source's spectral distributions and the resulting coherence properties. Coherence length, is the measure of acceptable displacement between two wavefronts in which interference between the two wavefronts can still occur. There are two main types of displacement, spatial and temporal, which are often used to characterize interfering wavefronts. Spatial displacement refers to allowable magnitude of shear between wavefronts and originates from the spatial extent of the source of the two interfering wavefronts. Temporal dispalcement refers to allowable time delay (or equivalently optical path length) between two wavefronts, common in unbalanced interferometers such as the Fizeau and naturally develops from the spectral distribution of the source. These two types of coherence can be treated independently. ${ }^{19}$ For now, spatial coherence will be ignored as the implementation of the spectrally controlled source directly effects temporal coherence propoerties and spatial coherence effects are of little consequence.
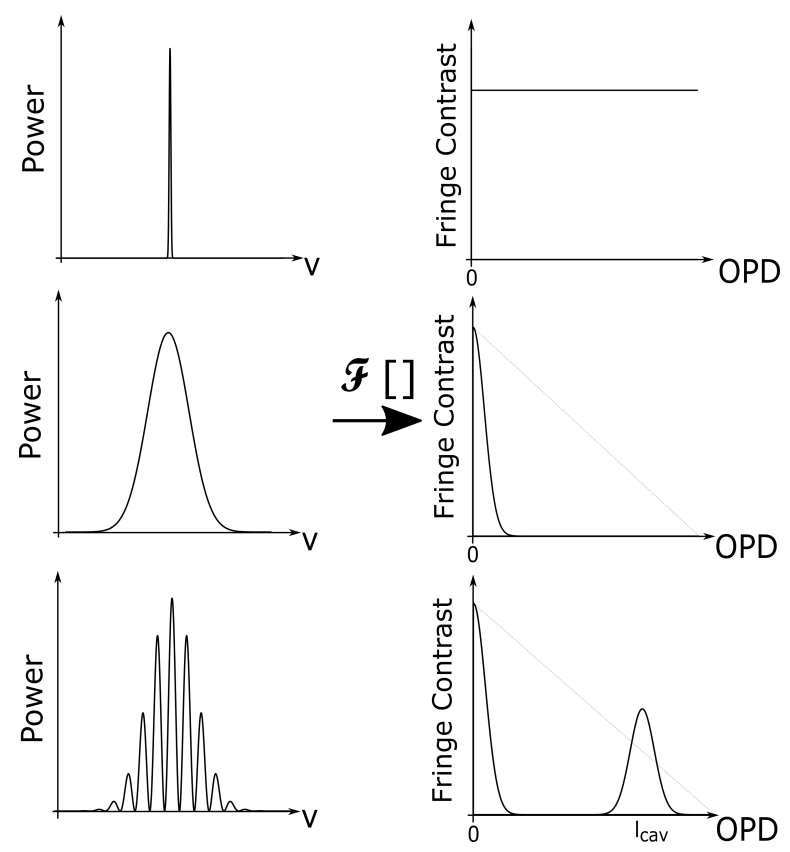

Figure 1. Spectral distributions (left) and resulting coherence functions (right) for laser source (top), broadband source (middle), and SCI source (bottom).

From the Wiener-Khinchin Theorem, the coherence function, $\gamma(\tau)$, which describes interference fringe contrast versus wavefront delay, of any optical source is related to the Fourier Transform, $\mathcal{F}[\bullet]$, of the power spectral density, $S(\nu)$, the modulus squared of the spectral distribution. The transform variable is, $\tau$, the time delay variable between two wavefronts. in units of seconds and follows the relationship to $(3), \tau=1 / \nu ., 19$

$$
\gamma(\tau)=\int_{-\infty}^{\infty} S(\nu) e^{2 \pi i \tau \nu} d \nu=\mathcal{F}[S(\nu)]
$$

Coherence length, $l_{c o h}$, is a characteristic length of the source at which fringe contrast falls below a nominal threshold of detection. Shown in (3), coherence length is a function of the optical frequency distribution where, $c$, is the speed of light and, $\Delta \nu$, is the optical frequnecy bandwidth assuming a normal distribution.

$$
l_{c o h}=\frac{c}{\Delta \nu}
$$

For laser interferometers, $\Delta \nu$, is very small, on the order of hundreds of megahertz which results in a coherence length of meters-allowing an unbalanced interferometer (Fizeau, for example) configuration. White light sources 
have large bandwidths, on the order of single terahertz, resulting in a coherence length of hundred of microns-forcing a balanced interferometer (Twyman-Green, for example) configuration. Figure 1 shows general spectral distributions on the left with corresponding coherence functions on the right for laser sources (top), broadband sources (middle), and SCI source (bottom).

The spectrally controlled source, as its name suggests, employs a higher level of control over the source's spectral distribution to enable tunable fringe localization as well as phase shifting. The source utilizes a nominally gaussian distribution broadband source, $g_{\text {nom }}(\nu ; \Delta \nu)$, similar to that of a white light or incoherent interferometer. From (2) and the previous discussion of white light interferomters, the resulting coherence function is also a Gaussian function, centered at $\tau=0$ whose temporal width is inversely proportional to the spectral width and coherence length follows from $(3) .{ }^{10}$

$$
g_{\text {complete }}=g_{\text {nom }} \times m(\nu ; f, \theta)=g_{\text {nom }} \times\left[\frac{1}{2}+\frac{1}{2} \cos (f \nu+\theta)\right]
$$

However, the nominal distribution is combined with a modulation function, $m(\nu ; f, \theta)$, resulting in a final source spectral distribution in (4). The modulation frequency, $f$, and modualtion function phase, $\theta$, are user controlled parameters. Via the Convolution Theorem, the coherence function of the complete SCI spectrum, $g_{\text {complete }}$, is given by (5), where $G_{n o m}$ is the Fourier transform of $g_{n o m}$ and $M(\nu ; f, \theta)$ is the Fourier transform of the modulation function, $m(\nu ; f, \theta)$. The final coherence function is normalized to achieve nominal fringe contrast of unity at $O P D=0 .{ }^{10}$

$$
\begin{aligned}
\gamma(\tau) & \propto G_{n o m} \otimes M(\nu ; f, \theta)=G_{n o m} \otimes\left[\frac{1}{2} \delta(\tau)+\frac{1}{4} e^{-i \theta} \delta(\tau-f)+\frac{1}{4} e^{i \theta} \delta(\tau+f)\right] \\
& =G(\tau)+\frac{1}{2} e^{-i \theta} G(\tau-f)+\frac{1}{2} e^{i \theta} G(\tau+f)
\end{aligned}
$$

In addition to the nominal coherence envelope located at $\tau=0$ in (5), there are two additional satellite coherence envelopes whose location are at non-zero $\tau$ locations. The locations of these sidebands are directly related to the modulation frequency, $f$, and the complex phase shift of the interference fringes at these locations are tied to phase of the modulation function, $\theta$ of the spectrally controlled source. The resulting scale factor at these coherence envelope locations limits the ideal SCI fringe contrast to 0.5. The location of these fringes can be rewritten in terms of the source mean wavelength, $\lambda_{0}$, and modulation period, $\Delta \lambda$, and in unit of distance (OPD) rather than in unit of time, shown in (6) to correspond to a designated cavity length, $l_{\text {cav }}$, or projected fringe distance. ${ }^{10}$

$$
l_{\text {cav }}=\frac{\lambda_{0}^{2}}{2 \Delta \lambda}
$$

With these two control parameters, the operater can localize fringes to single surfaces, also denoted in Figure 1 as $l_{c a v}$, and and then phase shift these fringes for complete PSI measurements without any mechanical mechanisms or translation.

Additionally, as with white-light interferometry, the width of these coherence envelopes is proportional to the bandwidth of $g_{n o m}$. However, this parameter can also be tuned with the spectrally controlled source to either increase or decrease the level of surface isolation required. The spectrally controlled source has been demonstrated to achieve measurements of 50.8 micron thin optical cavities. ${ }^{9}$

\section{MEASUREMENT AND RESULTS}

Multiple surface cavities are common measurement configurations for both surface figure measurements, as well as refractive index homogeneity measurements and provide significant challenges for both coherent and incoherent measurement systems. However, the spectrally controlled source is well suited for these applications and the measurement of planar optics. ${ }^{9-11}$ 


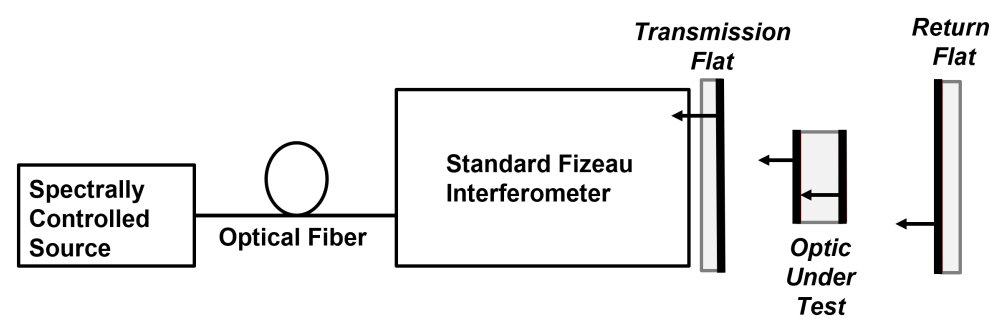

Figure 2. Experimental diagrams for refractive index homogeneity measurement with Fizeau interferometer upgraded with fiber coupled SCI Source. Each cavity for the four required measurements are isolated individually corresponding to the proper tuning of the source.

From an implementation standpoint, the spectrally controlled source has the advantage of employing all control over fringe location, working distance, and phase at the source level. As a result, any standard interferometer can be ugraded to be an SCI capable instrument with no significant modifications to the instrument.

An Äpre Instruments S100 - HR coherent Fizeau interferometer was integrated with a fiber coupled spectrally controlled source for the measurement of a $5 \mathrm{~mm}$ thick, $50 \mathrm{~mm}$ diameter Rolyn plane parallel plate was used as a test piece. This window was mounted in the measurement space between the transmission flat of the instrument and the return flat, shown in Figure 2.

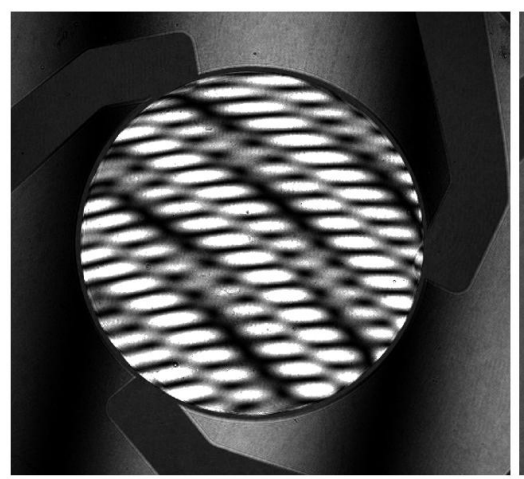

(a)

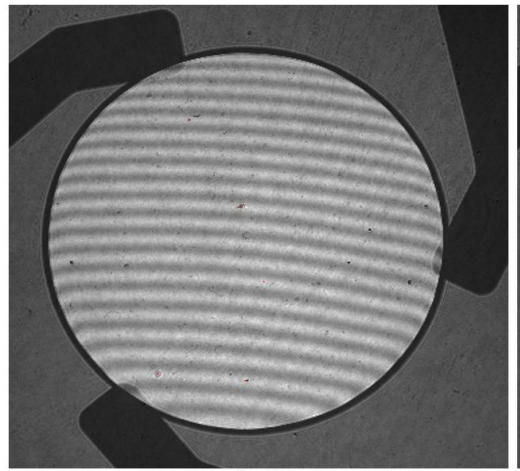

(c)

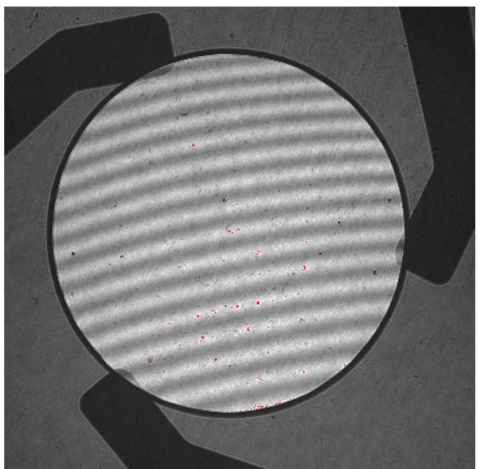

(b)

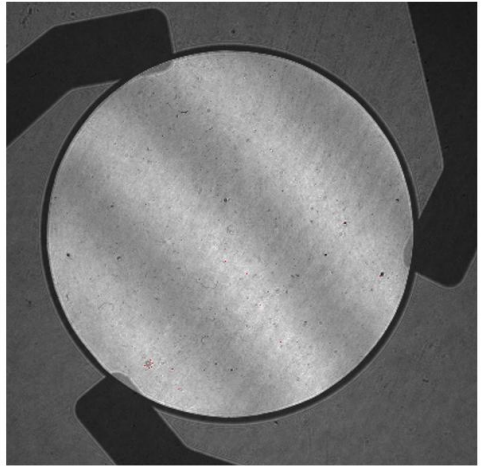

(d)

Figure 3. Various accessible interferograms with Fizeau interferometer upgraded with spectrally controlled source. (a) Coherent interferogram for spectrally controlled source in alignment mode. (b) Front surface cavity (c) Back surface cavity (d) Transmitted wavefront cavity (transmission flat-reference flat).

As mentioned in Section 1, the largest advantage of coherent interferometers is their ease of use in alignment of test surfaces and the high contrast fringe feedback regardless of axial location of the optic under test. A 


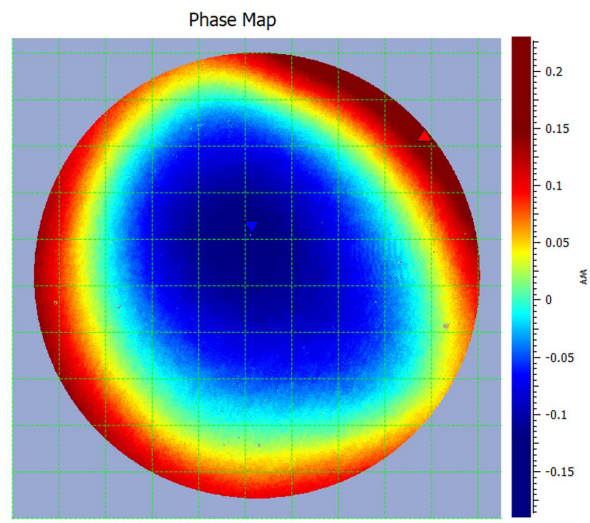

(a)

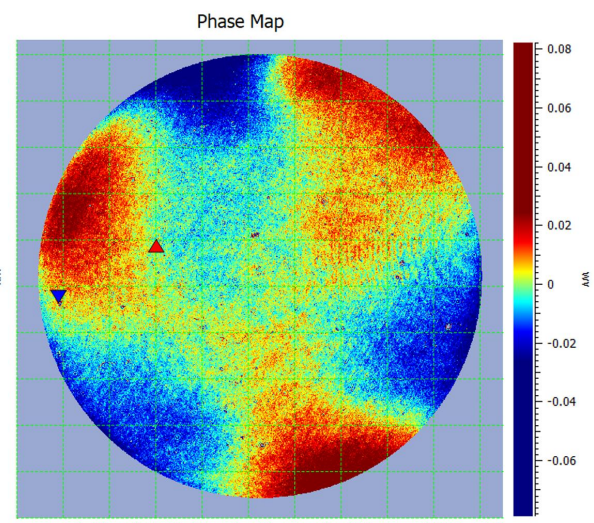

(b)

Figure 4. Front surface surface figure PSI measurement results from Fizeau interferometer upgraded with spectrally controlled source. (a) phase map with Zernike Tilt removed (b) phase map with low order Zernike terms removed

practical advantage of the spectrally controlled source is the flexibility in source bandwidth. While the operating condition for measurement is to produce localized fringes with a nominally broadband spectrum, the source can also be tuned to operate in a narrow bandwidth mode for a coherent alignment mode operating condition. This allows the operator to align any number of surfaces to the interferometer simulatensously and then switch to measurement mode for surface isolation and fringe localization. ${ }^{11}$ For measurement, the source is tuned to produce fringes at the distance which correponds to each individual cavity, realizing a single interferogram for each cavity. Various SCI interferograms for the experimental setup are shown in in Figure 3. Interferogram (a) is the coherent mode operation for simultaneous alignment of the four surface cavity. Interferogram (b) corresponds to the front surface cavity. Interferogram (c) corresponds to the back surface cavity. Interferogram (d) correpsonds to the transmitted wavefront cavity. The empty cavity interferogram has been omitted for conciseness as it is used for calibration of the reference surfaces. This measurement provides little insight into the behavior of the spectrally controlled source and is not unique to the SCI method.

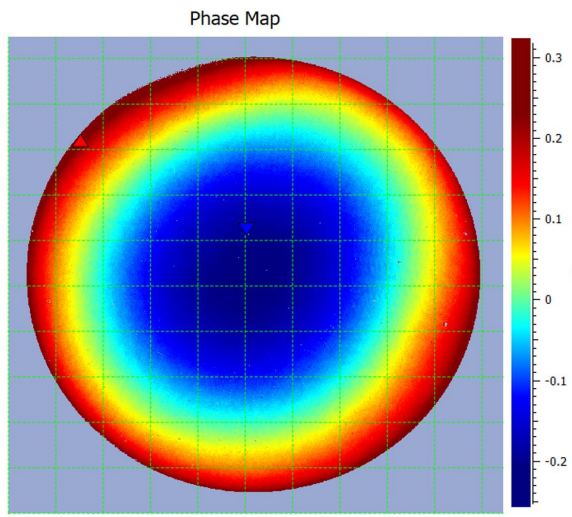

(a)

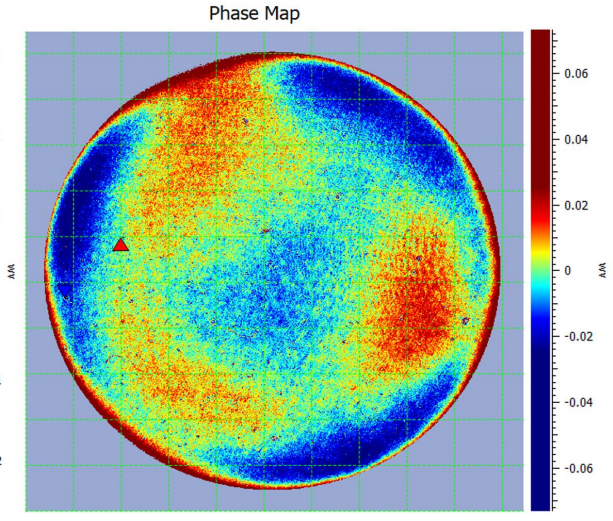

(b)

Figure 5. Back surface surface figure PSI measurement results from Fizeau interferometer upgraded with spectrally controlled source. (a) phase map with Zernike Tilt removed (b) phase map with low order Zernike terms removed

A complete PSI measurement and corresponding phase map is acquired for each cavity and averagd over five measurements. Figure 4, shows the phase map (a) with Zernike Tilt removed and the residual phase map (b) with low order Zernike terms removed including Tilt, Power, Spherical Aberration, Coma, and Astigmatism to show the underlying structure and the quality of measurements from the SCI Fizeau. The reported Peak-Valley for the phase map (a) is 0.413 waves and the repored RMS value is 0.074 waves. For the residual phase map (b), 
the reported Peak-Valley value is 0.161 waves and the reported RMS is 0.012 waves.

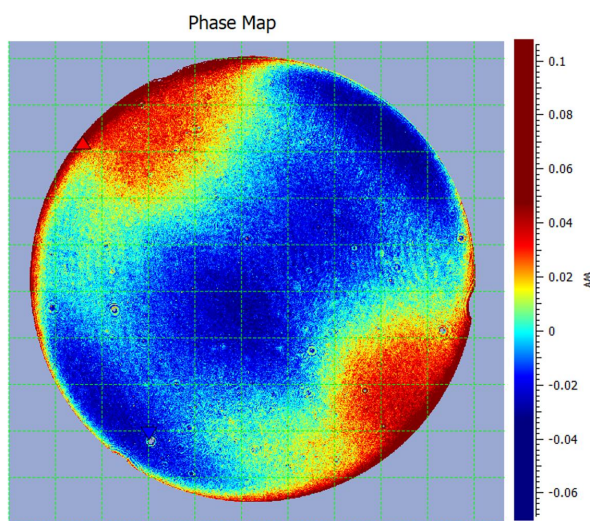

(a)

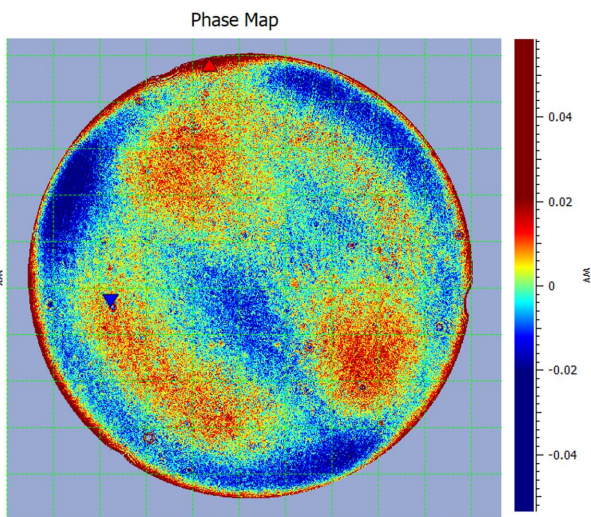

(b)

Figure 6. Transmitted wavefront (transmission flat-reference flat) surface figure PSI measurement results from Fizeau interferometer upgraded with spectrally controlled source. (a) phase map with Zernike Tilt removed (b) phase map with low order Zernike terms removed

Figure 5, shows the phase map (a) with Zernike Tilt removed and the residual phase map (b) with low order Zernike terms removed including Tilt, Power, Spherical Aberration, Coma, and Astigmatism to show the underlying structure and the quality of measurements from the SCI Fizeau. The reported Peak-Valley for the phase map (a) is 0.572 waves and the repored RMS value is 0.122 waves. For the residual phase map (b), the reported Peak-Valley value is 0.147 waves and the reported RMS is 0.012 waves.

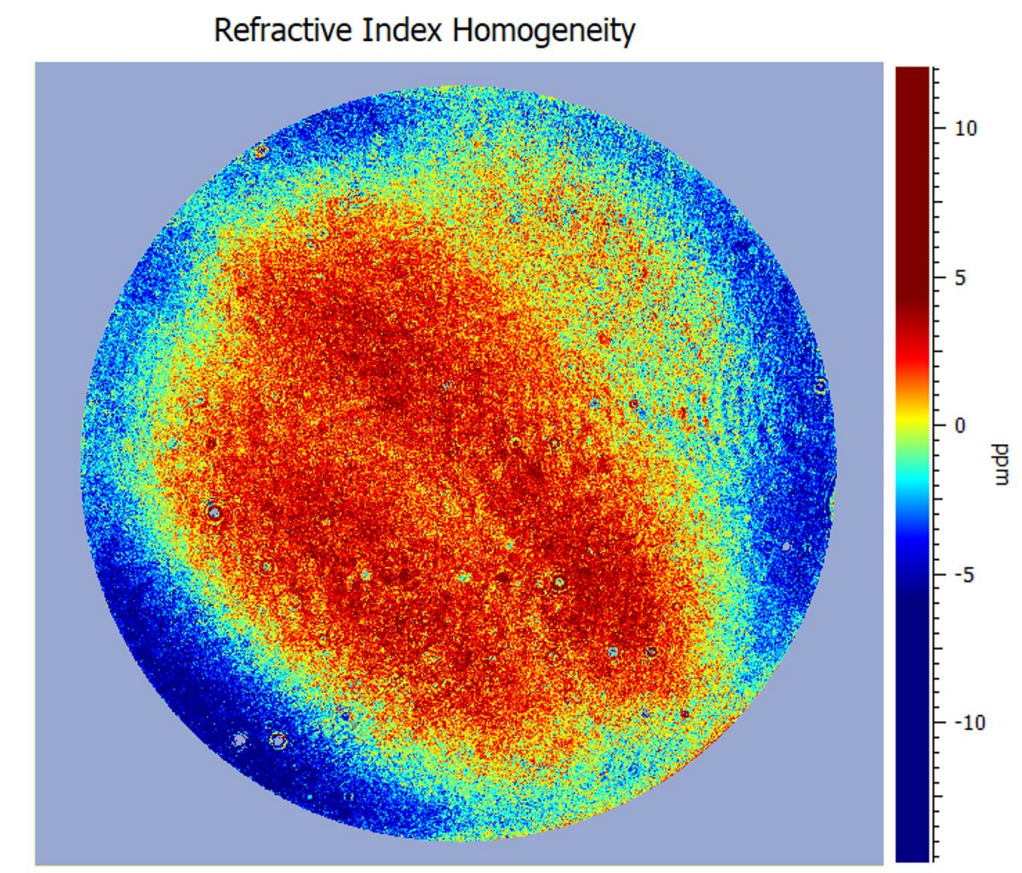

Figure 7. Processed refractive index homogeneity measurement result from Äpre Instruments metrology software, Reveal TM . The reported RMS value is 2.646 parts per million $\left(n_{0} \pm .000002646\right)$; reported Peak-Valley is 26.749 ppm.

Figure 6, shows the phase map (a) with Zernike Tilt removed and the residual phase map (b) with low order Zernike terms removed including Tilt, Power, Spherical Aberration, Coma, and Astigmatism to show the underlying structure and the quality of measurements from the SCI Fizeau. The reported Peak-Valley for the 
phase map (a) is 0.175 waves and the repored RMS value is 0.021 waves. For the residual phase map (b), the reported Peak-Valley value is 0.112 waves and the reported RMS is 0.010 waves.

With the acquired phase maps (a) in Figures 4-6 and an additional phase map for the measured empty cavity, refractive index homogeneity can be calculated based on the procedure described in Section 1.2 and the relationship in (1). ${ }^{18}$ The results were processed in Äpre Instruments metrology software, Reveal ${ }^{\mathrm{TM}}$. Figure 7, shows the resulting refractive index homogeneity map for the plane parallel plate in units of partper-million (ppm) of refractive index such that the values can be interpreted as $n_{0}+n \times 10^{-6}$, where $\mathrm{n}$ is the local homogeneity value. The reported Peak-Valley for this measurement is $26.749 \mathrm{ppm}$ and the reported RMS is $2.646 \mathrm{ppm}$.

\section{CONCLUSIONS}

The spectrally controlled source is an enabling approach to optical surface isolation and interferomtric measurement of multiple surface cavities which overcomes the inherent limitations of coherent, laser interferometer systems while providing a much more flexible and low complexity alternative to incoherent, white light interferometers. While fringe contrast for this method is limited to a nominal value of 0.5 , quality of commercial pahse shifting algorithms and the availability of 12-bit and 16-bit detectors prevent this from becoming a barrier to measurement.

Via control over the spectral modulation frequency, fringes can be localized at tunable locations removing the necessity for mechanically based, translating reference arms which require high level of precision, calibration, and add significant cost and complexity to interferometer systems. Control over modulation function phase allows localized fringes to be phase shifted without mechanical means. As all control parameters are implemented at the source level, the spectrally controlled source is a good candidate for upgrading existing interferometer systems with little modification and at lower cost.

Multiple cavity measurements with an SCI have been demonstrated to achieve effective surface isolation with no modification to the optic under test at high levels of accuracy regularly achieving measurements with RMS values at the $\lambda / 50$ level and down to the level of $\lambda / 100$.

\section{ACKNOWLEDGMENTS}

The authors acknowledge the University of Arizona College of Optical Sciences for supplying the planar optical window for measurement and testing.

\section{REFERENCES}

[1] M. Born and E. Wolf, Principles of Optics: Electromagnetic Theory of Propagation, Interference and Diffraction of Light, Cambridge, 7 ed., 1980.

[2] K. Creath, "V Phase-Measurement Interferometry Techniques," Prog. Opt. 26(C), pp. 349-393, 1988.

[3] C. Ai and J. C. Wyant, "Effect of spurious reflection on phase shift interferometry," Appl. Opt. 27, p. 3039, jul 1988.

[4] M. Trusiak and K. Patorski, "Two-shot fringe pattern phase-amplitude demodulation using Gram-Schmidt orthonormalization with Hilbert-Huang pre-filtering, " 2015.

[5] M. Trusiak, Ł. Służewski, and K. Patorski, "Single shot fringe pattern phase demodulation using HilbertHuang transform aided by the principal component analysis ," 2016.

[6] J. C. Wyant, "White light interferometry," 4737, pp. 98-107, International Society for Optics and Photonics, jul 2002.

[7] K. Okada, H. Sakuta, T. Ose, and J. Tsujiuchi, "Separate measurements of surface shapes and refractive index inhomogeneity of an optical element using tunable-source phase shifting interferometry.," Appl. Opt. 29(22), pp. 3280-5, 1990.

[8] M. Suematsu and M. Takeda, "Wavelength-shift interferometry for distance measurements using the Fourier transform technique for fringe analysis.," Appl. Opt. 30(28), pp. 4046-55, 1991. 
[9] C. Salsbury and A. Olszak, "Thin Optical Window Measurement with a Spectrally Controlled Interferometer," in Am. Soc. Precis. Eng. Top. Meet., American Society of Precision Engineers, (Tucson), 2017.

[10] C. Salsbury and A. Olszak, "Spectrally controlled interferometry," Appl. Opt. 56(28), 2017.

[11] C. Salsbury and A. G. Olszak, "Spectrally controlled interferometry for measurements of flat and spherical optics," 10448, pp. 104481C-10448-7, 2017.

[12] I. E. Kozhevatov and E. K. Kulikova, "Interferometric Methods for Surface Testing. High-Order White-Light Interferometer," Instruments Exp. Tech. 44(1), pp. 84-87, 2001.

[13] I. E. Kozhevatov, E. A. Rudenchik, N. P. Cheragin, and E. H. Kulikova, "A new in situ method for testing the optical thickness of removed transparent elements," p. 50, International Society for Optics and Photonics, sep 2003.

[14] I. E. Kozhevatov and E. K. Kulikova, "Higher-order Wideband Optical Interferometry," Radiophys. Quantum Electron. 46(1), 2003.

[15] J. Schwider, "White-light Fizeau interferometer," Appl. Opt. 36(7), pp. 1433-1437, 1997.

[16] J. Schwider, "Coarse frequency comb interferometry," in Interferom. XIV Tech. Anal., J. Schmit, K. Creath, and C. E. Towers, eds., p. 706304, International Society for Optics and Photonics, aug 2008.

[17] D. Malacara, Optical Shop Testing: Third Edition, Wiley series in pure and applied optics, John Wiley \& Sons, Inc., Hoboken, NJ, USA, jun 2006.

[18] J. Schwider, R. Burow, K.-E. Elssner, R. Spolaczyk, and J. Grzanna, "Homogeneity testing by phase sampling interferometry," Appl. Opt. 24(18), pp. 3059-3061, 1985.

[19] H. H. Barrett and K. J. Myers, "Foundations of Image Science ," 2013. 